\title{
A New Editing Scheme Based on a Fast Two-String Median Computation Applied to OCR
}

\author{
José Ignacio Abreu Salas ${ }^{1}$ and Juan Ramón Rico-Juan ${ }^{2}$ \\ ${ }^{1}$ Universidad de Matanzas, Cuba \\ jose.abreu@umcc.cu \\ 2 Dpto Lenguajes y Sistemas Informáticos, Universidad de Alicante, Spain \\ juanra@dlsi.ua.es
}

\begin{abstract}
This paper presents a new fast algorithm to compute an approximation to the median between two strings of characters representing a 2D shape and its application to a new classification scheme to decrease its error rate. The median string results from the application of certain edit operations from the minimum cost edit sequence to one of the original strings. The new dataset editing scheme relaxes the criterion to delete instances proposed by the Wilson Editing Procedure. In practice, not all instances misclassified by its near neighbors are pruned. Instead, an artificial instance is added to the dataset expecting to successfully classify the instance on the future. The new artificial instance is the median from the misclassified sample and its same-class nearest neighbor. The experiments over two widely used datasets of handwritten characters show this preprocessing scheme can reduce the classification error in about $78 \%$ of trials.
\end{abstract}

\section{Introduction}

Dataset editing has received considerable attention from the seminal works of Wilson [17] about the edited near neighbor rule (ENN) because this technique can be useful to improve nearest neighbor classifiers response. Mainly, these algorithms focus on deleting wrong tagged instances from a set, which will be used as training set for a given classifier. Several modifications have been proposed such as [2][4] [13] and [15][18] more recently, facing with some problems of basic Wilson procedure as statistically dependence of estimations over each instance [10]. Another group of algorithms also changes some instances tag while editing, such [6] and [14].

In many problems, patterns do not have a vectorial representation, instead another syntactic coding such strings and trees are commonly used. Methods cited before mainly concern with these vectorial representations and distances. Therefore, when dealing with strings a suitable distance has be selected. In our case, the widely used Levenshtein edit distance [7] is choosen. In addition, some approaches find representatives instances as centroids [12] or prototypes, so this concepts need to be extended to the new coding schemes.

Several authors [1] [3] [5] have described algorithms to get a prototype representing a set of strings, as the centroid, the median string or an approximation. Most of this works build the desired string by successive refinements of a initial string or by some ad-hoc procedure.

E.R. Hancock et al. (Eds.): SSPR \& SPR 2010, LNCS 6218, pp. 748-756, 2010.

(C) Springer-Verlag Berlin Heidelberg 2010 
This work proposes new Wilson based approach to edit a dataset of instances that have been encoded by some string representation. The inclusion of a prototype representing both, an instance and its same-class nearest neighbor inside a k-neighborhood if exist, when the instance is misclassified, is the main difference from others described in the literature. Besides, this paper presents a fast algorithm to compute the prototype representing two strings suitable for requirements of described editing procedure. Section 2 provides a detailed explanation of the algorithm to build the prototype and some useful concepts. Section 3 describes the proposed editing procedure and several considerations related to the computational complexity of proposed algorithms. Finally, section 4 illustrates with different experiments the behavior of proposed methods.

\section{Prototype Construction}

To compute the prototype representing two strings, this case defined as the median string, the proposed approach focus on information gathered from calculation of the distance between those strings. This section contains a glance of the selected distance measure, the Levenshtein [7] edit distance. Latter a procedure to compute the median string is covered.

\subsection{Edit Distance}

Let $\Sigma$ be an alphabet and $S_{1}=\left\{S_{11}, S_{12} . . S_{1 m}\right\}, S_{2}=\left\{S_{21}, S_{22} . . S_{2 n}\right\}$ two strings over $\Sigma$ where $m, n \geq 0$, the edit distance between $S_{1}$ and $S_{2}, D\left(S_{1}, S_{2}\right)$, is defined in terms of elementary edit operations which are required to transform $S_{1}$ into $S_{2}$. Usually three edit operations are considered:

- substitution of a symbol $a \in S_{1}$ by a symbol $b \in S_{2}$, denoted as $w(a, b)$

- insertion of a symbol $b \in S_{2}$ in $S_{1}$, denoted as $w(\varepsilon, b)$

- deletion of a symbol $a \in S_{1}$, denoted as $w(a, \varepsilon)$.

where $\varepsilon$ denotes an empty string. Let $Q_{S i}^{S j}=\left\{q_{1}, q_{2}, \ldots, q_{k}\right\}$ be a sequence of edit operations transforming $S_{i}$ into $S_{j}$, if each operation has cost $e\left(q_{i}\right)$ the cost of $Q_{S 2}^{S 1}$ is $E_{Q_{S 2}^{S 1}}=\sum_{i=1}^{k} e\left(q_{i}\right)$ and the edit distance $D\left(S_{1}, S_{2}\right)$ is defined as:

$$
D\left(S_{1}, S_{2}\right)=\operatorname{argmin}\left\{E_{Q_{S 2}^{S 1}}\right\} .
$$

Strings involved in this work are Freeman Chaincodes, for that reason substitution costs are computed as follows:

$$
e(w(a, b))=\operatorname{argmin}\{|a-b|, 8-|a-b|\}
$$

In the case of the insertions and deletions the cost 2 was chosen which is half of the maximum cost of the substitution operation; this same fixed number is used in [11]. The dynamic programming algorithm exposed by Wagner [16] allows to compute $D\left(S_{1}, S_{2}\right)$ in $\mathcal{O}\left(L_{S 1} \times L_{S 2}\right)$ time, where $L_{S}$ denotes the length of string $S$. 


\subsection{Fast Median String Computation}

The median of a set $T$ of strings can be briefly defined as the string $R$ which realizes:

$$
\operatorname{argmin}_{R}\left\{\sum D\left(R, S_{i}\right) \mid S_{i} \in T\right\}
$$

As explained above, the proposed approach computes the median $R$ of two strings $S_{1}$ and $S_{2}$ by applying a subset of edit operations from the minimum cost edit sequence $Q_{S 2}^{S 1}$ to $S_{1}$. To choose those editions that will be applied, each element at $Q_{S 2}^{S 1}$ is tested to estimate how it will affect $D\left(S_{1}, R\right)$ and $D\left(S_{2}, R\right)$, since the algorithm seeks for a string $R$ satisfying (1) and (2). This additional requirement means that an $R$ near of the halfway between $S_{1}$ and $S_{2}$ will be preferred.

$$
\operatorname{argmin}_{R}\left\{\left|D\left(R, S_{1}\right)-D\left(R, S_{2}\right)\right|\right\}
$$

The idea behind the algorithm is that each operation $q_{i}$ in $Q_{S 2}^{S 1}$ affects the future $D\left(R, S_{1}\right)$ and $D\left(R, S_{2}\right)$ since can be guessed that a rejected operation keeps $R$ similar to $S_{1}$ while accepted editions makes $R$ resembles to $S_{2}$. A close examination of each possible operation help to explain this conjecture.

For insertions, let $b_{S 2}^{k}$ be the k-esime symbol from $S_{2}$. An operation $q_{i}=w\left(\varepsilon, b_{S 2}^{k}\right)$ from $Q_{S_{1}}^{S 2}$ indicates the insertion of this symbol into $S_{1}$ to obtain $R$. Suppose $q_{i}$ is accepted, thus can be expected that $Q_{R}^{S 2}$ does not involves an insertion of a symbol in $R$ to be matched with $b_{S 2}^{k}$ in $S_{2}$ since it was done before. A similar reasoning led to guess this symbol is market to deletion from $R$ when $D\left(R, S_{1}\right)$ is computed. In turn, a symbol $b_{S_{1}}^{k}$ deleted from $S_{1}$ to get $R$ will be pointed to be inserted again while computing distance from $R$ to $S_{1}$.

Substitutions $w\left(b_{S_{1}}, b_{S_{2}}\right)$ will always applied, but whenever possible a symbol $m$ will be placed in $R$ instead $b_{S_{1}}$ or $b_{S_{2}}$. The choice of $m$ tries to make $R$ similar to both $S_{1}$ and $S_{2}$, thus must satisfies:

$$
\begin{gathered}
e\left(w\left(b_{S_{1}}, b_{S_{2}}\right)\right)=e\left(w\left(b_{S_{1}}, m\right)\right)+e\left(w\left(m, b_{S_{2}}\right)\right) . \\
\operatorname{argmin}_{m}\left\{\left|e\left(w\left(m, b_{S_{1}}\right)\right)-e\left(w\left(m, b_{S_{2}}\right)\right)\right|\right\} .
\end{gathered}
$$

Previous assumptions allow estimating how applying or not an operation $q_{i}$ will affect distances from $R$ to $S_{1}$ and $S_{2}$. Chosen insertions of $b_{S 2}$ into $S_{1}$ contributes with $e\left(w\left(b_{S 2}, \varepsilon\right)\right)$ to $D\left(R, S_{1}\right)$ since the inserted symbol need to be deleted, now $q_{i}$ has not an effect on $D\left(R, S_{2}\right)$. If the insertion is rejected implies $D\left(R, S_{1}\right)$ does not change, but $D\left(R, S_{2}\right)$ will grow by $e\left(w\left(\varepsilon, b_{S 2}\right)\right)$. Unlike the insertion occurs in the case of the deletion operation. If a deletion is discarded $D\left(R, S_{2}\right)$ increases by $e\left(w\left(b_{S 1}, \varepsilon\right)\right)$ or $D\left(R, S_{1}\right)$ by $e\left(w\left(\varepsilon, b_{S 1}\right)\right.$, if operation is accepted. Substitutions make both $D\left(R, S_{1}\right)$ and $D\left(R, S_{2}\right)$ grow by $e\left(w\left(b_{S 1}, m\right)\right)$ and $e\left(w\left(m, b_{S 2}\right)\right)$ respectively.

For example, let $S_{1}=\{a, b\}, S_{2}=\{d, e\}$ and $e(w(\cdot, \varepsilon))=e(w(\varepsilon, \cdot))=1$. Table 1 shows the substitution cost between symbols, thus $Q_{S 1}^{S 2}=\{w(a, \varepsilon), w(b, d), w(\varepsilon, e)\}$. Possible options to select or not an operation yields the tree at Figure 1 where each leaf node shows a candidate $R$. Inside brackets, an estimation of the cumulative contribution of each operation up from the node to $D(R, S 1)$ and $D(R, S 2)$ respectively. Procedures 
FMSC and Find Op outlined below allow searching through the tree for those edit operations which yields an $R$ satisfying established requirements.

\section{Let:}

$Q_{S 1}^{S 2}$ :minimum cost edit sequence to transform $S_{1}$ into $S_{2}$.

$d$ : difference between cumulative $S 1$ and $S 2$.

$r$ : the better consecutive symbols corresponding to $d$ difference.

function FindOp $\left(o p_{i}, a_{S 1}, a_{S 2}\right):(d, r)$

/* $o p_{i}$ : index of the operation op $\in Q_{S 1}^{S 2}$ to analyze if is applied or not */

/* $a_{S 1}=0$ : cumulative distance of applied editions over $D(R, S 1) * /$

/* $a_{S 2}=0$ : cumulative distance of applied editions over $D(R, S 2) * /$

/* better $=(\infty, \emptyset)$ : local better result */

if $\left(o p_{i}==0\right)$ then

better $=\left(a_{S 1}-a_{S 2}, \emptyset\right)$

else

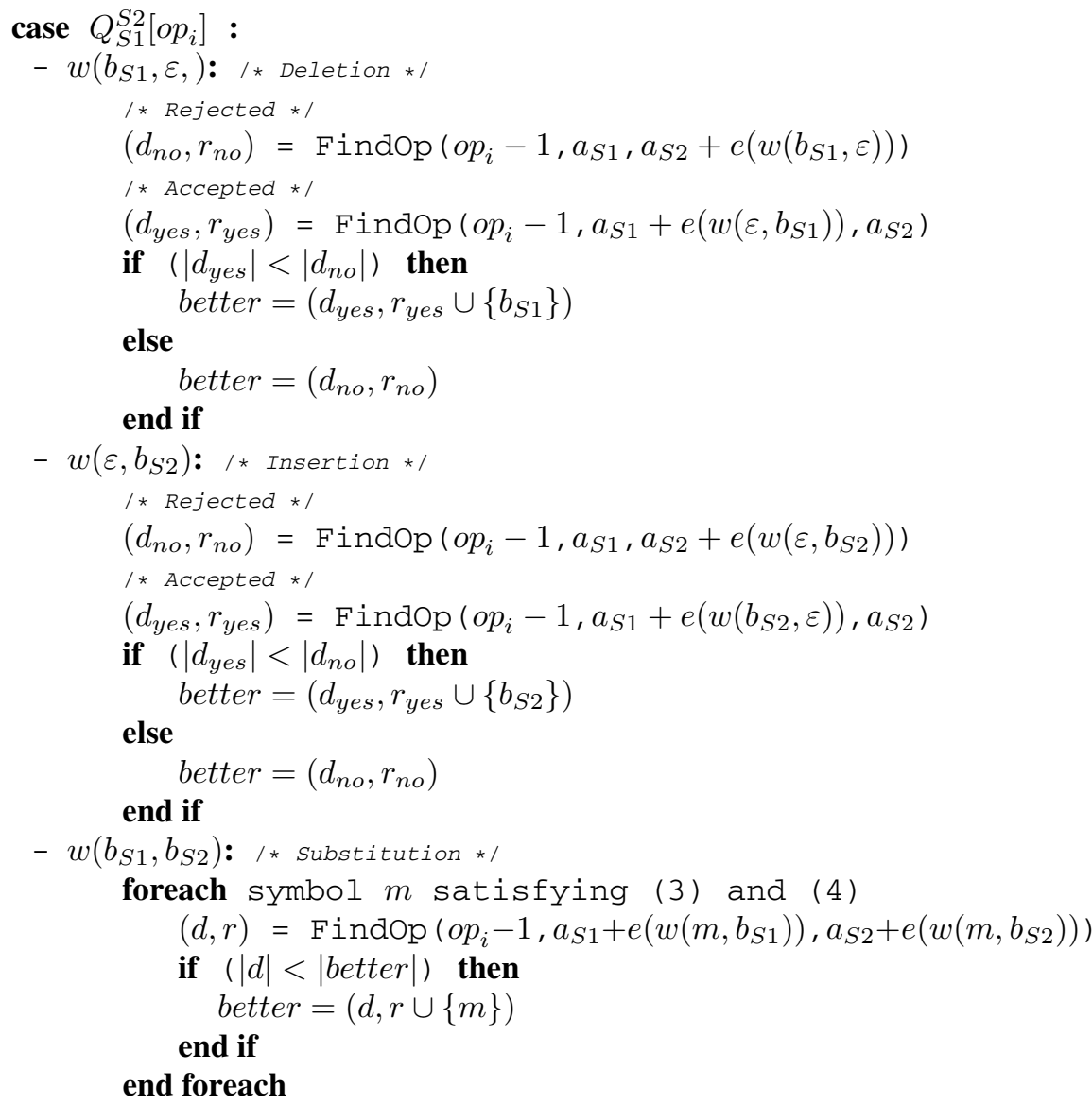

end case 


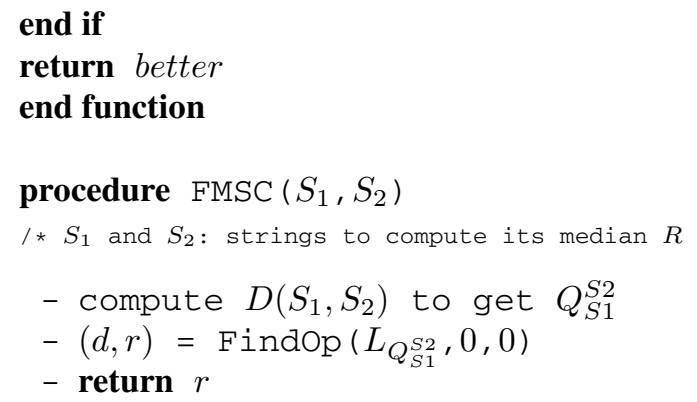

end procedure

Table 1. Substitution cost between two symbols. $e(w(\cdot, \cdot))$
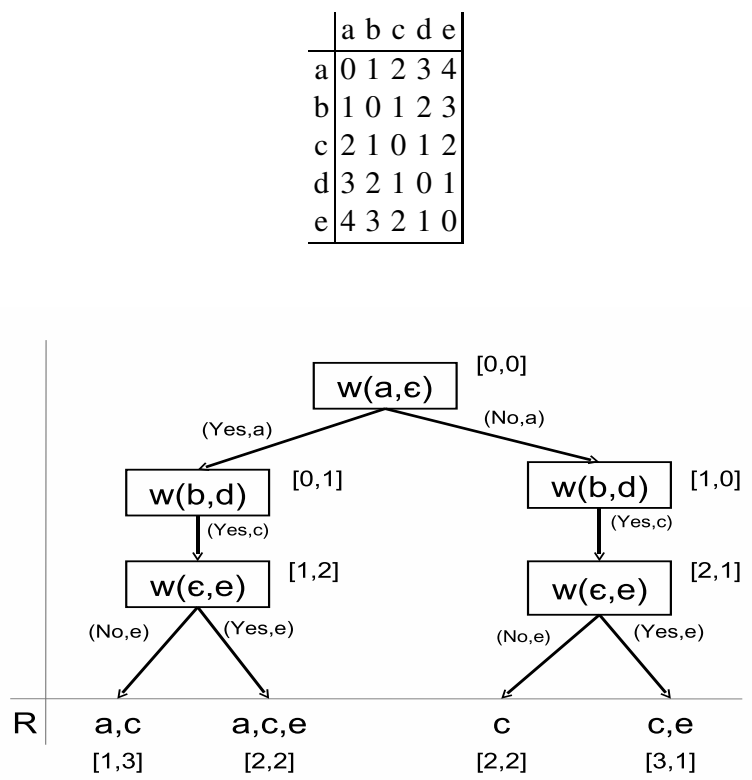

Fig. 1. Each branch represents a possible set of operations to get $R$ from $S_{1}$

\section{Editing Algorithm}

Let $T$ a set of instances. Wilson [17] based editing procedures such [4][13] remove all misclassified instances, $t_{i}$, by its $k$-nearest neighbors $(K-\mathrm{NN})$. This kind of editing cleans interclass overlapping regions while the boundaries between classes are smoothed. A $K$-NN classifier that uses the edited set as training set could be improved its classification results respect the classification through the original dataset.

As point Wilson [18], in some cases editing has be done carefully because the algorithm may remove a lot of instances, while spoiling the generalization capabilities. 
When $k \geq 1$, a wrong classification of $t_{i}$ does not mean that no one $\mathrm{k}$-nearest neighbors belongs to the same class of $t_{i}$. Thus, it is reasonable to think that $t_{i}$ does not need to be an outlier, but can be a boundary instance useful for next classifications.

The proposed approach aims to face successfully this problem by adding to $T$ an artificial instance $R$ computed from $t_{i}$ and its same-class nearest neighbor, $t_{j}$, if it belongs to $k$-nearest neighbors. If $R$, tagged as $t_{i}$, satisfies $D\left(R, t_{i}\right) \leq D\left(t_{i}, t_{j}\right)$ and (2). Its inclusion boosts the chance $t_{i}$ will be correctly reclassified since by definition $R$ lies in the $t_{i} \mathrm{k}$-neighborhood. Moreover, this can be viewed as some space regions poorly covered from the original instances become better represented. From these assumptions can be guessed this editing scheme leads to lower classification errors versus the original dataset. Artificial instance $R$ will be computed by the procedure Fast Median String Computation, FMSC for short, described at section 2.2, as $R=F M S C\left(t_{i}, t_{j}\right)$. The algorithm sketched below allows compute the edited set.

\section{Let:}

$T$ :instance set to edit.

$K$ :number of near neighbors.

foreach instance $t_{i}$ in $T$

- classify $t_{i}$ by its k-near neighbors in $T-t_{i}$. if wrong classified then

- find $t_{j}$, the $\mathrm{k}$-near same-class neighbor of $t_{i}$. if exist $t_{j}$ then

- build $R=F M S C\left(t_{i}, t_{j}\right)$.

- make $T=T \cup R$.

else

- mark $t_{i}$ to deletion.

\section{end if}

end if

\section{end foreach}

- delete from $T$ all market instances.

\subsection{Computational Cost Analysis}

Computing the median $R$ from strings $S_{1}$ and $S_{2}$ involves the calculation of $D\left(S_{1}, S_{2}\right)$, which can be accomplished in $\mathcal{O}\left(L_{S 1} \times L_{S 2}\right)$ as was pointed at subsection 2.1. From definition of this distance $L_{Q_{S 1}^{S 2}}=L_{S 1}+L_{S 2}$ for the worst case, i.e when there are no substitutions.

Searching through the tree with FindOp can be viewed as evaluating all possibilities to assigning $\{$ accepted/rejected $\}$ to every $q_{i}$ in $Q_{S 1}^{S 2}$, so there are $2^{L_{Q_{S 1}^{S 2}}}$ chances, this is, the number of branches on the tree. Denoting a rejected operation by $\sim q_{i}$, let $O p=\left\{q_{1}, \sim q_{2}, \sim q_{3}, \ldots, q_{n}\right\}$ be a possible assignation and $q_{i}, i<n$, an arbitrary operation. Clearly, the estimate $a_{S 1}$ to $D\left(R, S_{1}\right)$ associated with $O p$ can be decomposed as 
$a_{S_{1}}=a_{S_{1}}^{0 . . i}+a_{S_{1}}^{i+1 . . n}$ where $a_{S_{1}}^{k . . h}$ denotes the cumulative contribution of those operations $q_{k}, . ., q_{h}$ to $a_{S 1}$, this holds also for $a_{S 2}$.

$\mathrm{Be} O p^{\prime}$ a new assignment derived from $O p$ by changing the $\{$ accepted/rejected $\}$ tag to some operations $\left\{q_{0}, . ., q_{i}\right\}$ while $\left\{q_{i+1}, . ., q_{n}\right\}$ gets unchanged. Consequently the value $a^{\prime}{ }_{S 1}$ related to $O p^{\prime}$ is $a^{\prime} S_{1}=a_{S_{1}}^{\prime 0 . . i}+a_{S_{1}}^{i+1 . . n}$, an expression which is partially calculated before, similarly can be computed $a_{S 2}^{\prime}$. Moreover, if those assignations to $\left\{q_{i+1}, . ., q_{n}\right\}$ minimizes $\left|a_{S_{1}}^{i+1 . . n}-a_{S_{2}}^{i+1 . . n}\right|$ thus, the optimal sequence of assignations is one which have $\left\{q_{i+1}, . ., q_{n}\right\}$ as subsequence.

Considerations above allows to speed up computation of Find $O p$ procedure by applying a dynamic programming approach leading a $\mathcal{O}\left(\max \left\{L_{S 1} \times L_{S 2}, L_{Q_{S 1}^{S 2}} \times D_{s}\right\}\right)$, where $D_{s}=D\left(S_{1}, S_{2}\right)$.

The editing procedure needs to classify every instance at $T$, which requires an $\mathcal{O}\left(|T|^{2}\right)$ time. A second steps involves computing FMSC for every wrong classified instance having a same-class k-near neighbor. The worst case, all $|T|$ instances will need to be processed, so this step entails $\mathcal{O}(|T| \times$ FindOp $)$ time.

\section{Experimental Results}

The behavior of the proposed algorithms was analyzed using two sets of strings (Freeman Chaincodes). Digits and character contours from the NIST 3 DATABASE with 26 and 10 classes respectively.

To evaluate the editing algorithm a sample of 80 instances per class was drawn and each set splits in 4-fold to use a crossvalidation technique. At a first stage, for a fixed value of $K$, all training sets were edited by the Wilson procedure and each test set classified by the $K$-NN rule using the respective edited set. Latter, each original training set was edited but this time by our proposed approach classifying again the test sets. As

Table 2. Average error rate (4-folds) as percent for classification with different edited sets. (Characters set).

\begin{tabular}{|c|c|c|c|c|c|c|c|c|c|c|c|c|c|c|c|c|c|}
\hline & \multicolumn{16}{|c|}{ K on Edition } \\
\hline & & \multicolumn{2}{|c|}{$\mathrm{K}=3$} & \multicolumn{2}{|c|}{$\mathrm{K}=5$} & \multicolumn{2}{|c|}{$\mathrm{K}=7$} & \multicolumn{2}{|c|}{$\mathrm{K}=9$} & \multicolumn{2}{|c|}{$\mathrm{K}=11$} & \multicolumn{2}{|c|}{$\mathrm{K}=13$} & \multicolumn{2}{|c|}{$\mathrm{K}=15$} & \multicolumn{2}{|c|}{$\mathrm{K}=17$} \\
\hline 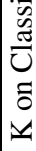 & 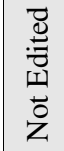 & $\begin{array}{l}\frac{0}{0} \\
\frac{0}{3} \\
\frac{3}{3}\end{array}$ & 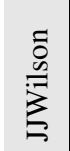 & $\begin{array}{l}0 \\
\frac{0}{3} \\
\frac{2}{3}\end{array}$ & 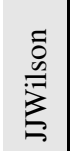 & 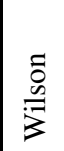 & 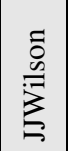 & 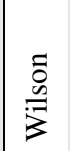 & 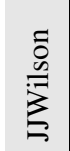 & 趸 & 总 & 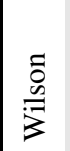 & 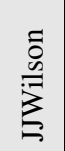 & $\begin{array}{l}\tilde{0} \\
0 \\
\stackrel{0}{3}\end{array}$ & 总 & $\frac{5}{0}$ & 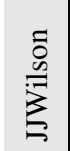 \\
\hline 1 & 13.7 & 16.5 & 14.6 & 15.8 & 13.8 & 16.3 & 13.8 & 16.8 & 13.8 & 17.1 & 13.4 & 17.1 & 13.4 & 17.5 & 13.2 & 17.8 & 13.1 \\
\hline 3 & 14.7 & 17.6 & 15.3 & 17.6 & 14.6 & 17.5 & 14.4 & 17.8 & 14.2 & 18.8 & 14.0 & 18.9 & 13.9 & 19.6 & 13.8 & 19.9 & 13.3 \\
\hline 5 & 15.4 & $\overline{17.6}$ & 15.2 & $\sqrt{17.9}$ & 14.4 & 17.9 & 14.1 & $\overline{18.5}$ & 14.1 & $\overline{18.9}$ & 14.2 & 19.5 & 14.1 & 19.8 & 13.8 & 20.2 & 13.7 \\
\hline 7 & 16.0 & 19.4 & 16.2 & 19.6 & 15.1 & \begin{tabular}{|l|}
19.9 \\
\end{tabular} & 15.2 & 19.8 & 14.7 & 20.2 & 14.2 & 20.7 & 14.0 & 20.8 & 14.0 & 21.3 & 14.1 \\
\hline 9 & 17.1 & 19.5 & 16.3 & 20.1 & 15.5 & 20.3 & 15.3 & 20.5 & 14.8 & 21.0 & 14.8 & 21.6 & 14.5 & 21.8 & 14.6 & 22.2 & 14.6 \\
\hline 11 & 17.7 & 20.0 & 17.7 & 20.8 & 16.5 & 20.8 & \begin{tabular}{|l|}
16.1 \\
\end{tabular} & 21.3 & 15.4 & 21.6 & 15.0 & 22.3 & 15.0 & 22.3 & 15.2 & 23.1 & 15.0 \\
\hline 13 & 18.3 & 21.0 & 18.2 & $\overline{21.2}$ & 17.1 & 21.7 & 16.4 & 22.1 & 16.5 & 22.5 & 15.8 & 22.8 & 15.5 & 23.3 & 15.5 & 23.7 & 15.7 \\
\hline 15 & 18.6 & 22.0 & 18.9 & 21.6 & 18.0 & 22.3 & \begin{tabular}{|l|}
17.1 \\
\end{tabular} & 22.6 & 16.7 & 23.3 & 16.2 & 23.5 & 16.3 & 23.7 & 16.0 & 24.2 & 16.0 \\
\hline 17 & 19.6 & 22.1 & 18.8 & 22.7 & 18.0 & 23.0 & 17.5 & 23.8 & 17.4 & 24.0 & 16.9 & 24.0 & 16.5 & 24.6 & 16.5 & 24.8 & 16.4 \\
\hline
\end{tabular}


Table 3. Average error rate (4-folds) as percent for classification with different edited sets. (Digits set).

\begin{tabular}{|c|c|c|c|c|c|c|c|c|c|c|c|c|c|c|c|c|}
\hline & \multicolumn{15}{|c|}{ K on Edition } \\
\hline & & \multicolumn{2}{|c|}{$\mathrm{K}=3$} & \multicolumn{2}{|c|}{$\mathrm{K}=5$} & \multicolumn{2}{|c|}{$\mathrm{K}=7$} & \multicolumn{2}{|c|}{$\mathrm{K}=9$} & \multicolumn{2}{|c|}{$\mathrm{K}=11$} & \multicolumn{2}{|c|}{$\mathrm{K}=13$} & \multicolumn{2}{|c|}{$\mathrm{K}=15$} & $\mathrm{~K}=17$ \\
\hline 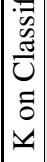 & \begin{tabular}{|l}
$:=$ \\
0 \\
iI \\
0 \\
Z \\
$z$
\end{tabular} & 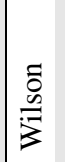 & 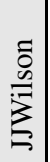 & 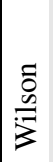 & $\begin{array}{l}0 \\
0 \\
0 \\
0 \\
0 \\
0\end{array}$ & $\begin{array}{l}0 \\
0 \\
0 \\
0 \\
3 \\
3\end{array}$ & $\begin{array}{l}0 \\
0 \\
0 \\
0 \\
0\end{array}$ & 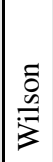 & 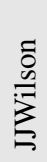 & $\begin{array}{l}0 \\
0 \\
0 \\
3 \\
3\end{array}$ & 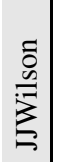 & $\begin{array}{l}0 \\
0 \\
0 \\
3 \\
3\end{array}$ & 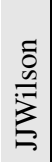 & 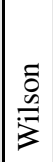 & 音 & $\begin{array}{l}0 \\
0 \\
0 \\
0 \\
0\end{array}$ \\
\hline 1 & 1.8 & $\bar{\Omega}$ & & 2 & 1.8 & 2.5 & 1.8 & $\sqrt{2.9}$ & 1.6 & 2.8 & 1.6 & 2.8 & 1.6 & 2.8 & 1.5 & 2.81 .5 \\
\hline & & 2 & & $\overline{2.9}$ & 2.3 & 3.0 & 2.0 & 3.3 & 1.9 & 3.4 & 2.0 & 3.4 & 2.0 & 3.8 & 1.9 & 3.91 .8 \\
\hline & 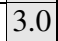 & 3.6 & 2.9 & 3.6 & 2.8 & 3.8 & 2.8 & 4.3 & 2.6 & 4.3 & 2.6 & 4.12 & 2.5 & 4.3 & 2.3 & 4.42 .5 \\
\hline 7 & 3.5 & 3 & 3.5 & 4.3 & 2.9 & 4.3 & 2.9 & 4.5 & 2.6 & 4.6 & 2.6 & 4.82 & 2.6 & 5.0 & 2.5 & 5.12 .4 \\
\hline & 3.6 & ? & 3.5 & $\sqrt{4.1}$ & 3.3 & 4.3 & 3.0 & 4 & 2.9 & 4.9 & 2.8 & $\overline{4.9}$ & 2.8 & |5.1 & 2.8 & 5.42 .8 \\
\hline & 4.1 & 72 & & 4 & & 4.6 & 2.9 & 4.6 & & 4.6 & 3.6 & & & 4.9 & 3.5 & 5.33 .4 \\
\hline & 4 & 3 & 3.1 & 4.8 & 3.3 & 5.0 & 3.1 & 5.0 & 3.3 & 5.3 & 4.0 & 5.6 & 3.5 & 5.9 & 3.5 & 5.93 .4 \\
\hline & 0 & & & & & 5.4 & 3.6 & & & 5.5 & 4.5 & & 4.3 & 16.3 & 4.1 & 6.33 .9 \\
\hline & 4.9 & 1 & 4.1 & 5.1 & 4.0 & 54 & 3.8 & & 3.9 & 5.5 & 4.6 & & 42 & & 43 & 5.14 .3 \\
\hline
\end{tabular}

a baseline, the original training sets classficantion is used. At each fold, editing was repeated for odds values of $K$ from 3 to 17, while in the classification stage, the range was from 1 to 17 . Remaining a total of 288 trials on each dataset. As distance, the Levenshtein distance was chosen which is described in the section 2.1.

Tables 2 and 3 show some results for the 4-fold experiments when the test set uses: the original training set, different edited sets for the characters and the digits datasets, respectively. Through these experiments, Wilson procedure never reduces the baseline error rate (classification with original tranining sets), while our proposed approach, labeled as JJWilson, is to able to improve by $79.1 \%$ of the trials in the case of characters dataset and by $77.7 \%$ in the case of digits dataset. These improvements are highlighted in bold type in the tables of results. So, the experiments in both datasets show that the proposed algorithm for editing outperforms the Wilson approach, with respect to the error rate reduction.

\section{Conclusions and Future Work}

A novelty method was presented to edit a dataset of contours encoded by Freeman Chaincodes. In addition, a new fast procedure to compute the median between two strings based on a string edit distance is explained. Experiments show that the edit scheme behaves well on the studied datasets. Further investigations can be addressed to revise the method to identify the misclassified instance, and consider other near neigbor belonging to the same class instead the nearest one to build the new prototype. Also, others datasets could be studied and compared with additional edit methods. Moreover, our fast median string algorithm between two examples could be extended to compute the average of $N$ examples. 


\section{Acknowledgements}

This work is partially supported by the Spanish CICYT under project DPI2006-15542C04-01, the Spanish MICINN through project TIN2009-14205-CO4-01 and by the Spanish research programme Consolider Ingenio 2010: MIPRCV (CSD2007-00018).

\section{References}

1. Cárdenas, R.: A Learning Model for Multiple-Prototype Classification of Strings. In: 17th International Conference on Pattern Recognition, vol. 4, pp. 420-442 (2004)

2. Devijver, I., Kittler, J.: On the edited nearest neighbour rule. In: 5th Int. Conf. on Pattern Recognition, pp. 72-80 (1980)

3. Duta, N., Jain, A., Dubuisson-Jolly, M.: Automatic Construction of 2D Shape Models. IEEE Transactions on Pattern Analysis and Machine Intelligence 23, 433-446 (2001)

4. Ferri, F., Vidal, E.: Comparison of several editing and condensing techniques for colour image segmentation and object location. Pattern Recognition and Image Analysis (1992)

5. Jiang, X., Schiffmann, L., Bunke, H.: Computation of median shapes. In: 4th Asian Conference on Computer Vision (2000)

6. Koplowitz, J., Brown, T.: On the relation of performance to editing in nearest neighbour rules. Pattern Recognition 13, 251-255 (1981)

7. Levenshtein, V.: Binary codes capable of correcting deletions, insertions and reversals. Soviet Physics 10, 707-710 (1966)

8. Martínez, C., Juan, A., Casacubierta, F.: Median strings for k-nearest neighbour classification*1. Pattern Recognition Letters 24, 173-181 (2003)

9. Olvera, J., Martínez, F.: Edition schemes based on BSE. In: 10th Iberoamerican Congress on Pattern Recognition, pp. 360-368 (2005)

10. Penrod, C., Wagner, T.: Another look at the edited neares neighbour rule. IEEE Trans. on Systems, Man and Cybernetics 7, 92-94 (1977)

11. Rico-Juan, J.R., Micó, L.: Comparison of AESA and LAESA search algorithms using string and tree-edit-distances. Pattern Recognition Letters 24, 1417-1426 (2003)

12. Sánchez, J., Pla, F., Ferri, F.: Using the nearest centroid neighbourhood concept for editing purposes. In: 7th Symposium National de Reconocimiento de Formas y Análisis de Imágen, vol. 1, pp. 175-180 (1997)

13. Tomek, I.: An experiment with the edit nearest neighbour. IEEE Trans. on Systems, Man and Cybernetics 6, 448-452 (1976)

14. Tomek, I.: A generalization of the k-NN rule. IEEE Trans. on Systems, Man and Cybernetics 6, 121-126 (1976)

15. Vázquez, F., Sánchez, J., Pla, F.: A stochastic approach to Wilson's editing algorithm. In: Marques, J.S., Pérez de la Blanca, N., Pina, P. (eds.) IbPRIA 2005. LNCS, vol. 3523, pp. 35-42. Springer, Heidelberg (2005)

16. Wagner, R., Fischer, M.: The String-to-String Correction Problem. Journal of the ACM 21, 168-173 (1974)

17. Wilson, D.: Asymptotic properties of nearest neighbor rules using edited data. IEEE Trans. on Systems, Man. and Cybernetics 2, 408-421 (1972)

18. Wilson, D., Martínez, T.: Reduction techniques for instance based learning algorithms. Machine Learning 38, 257-286 (2000) 